\title{
Solitary waves on nonlinear elastic rods. I
}

\section{Sørensen, Mads Peter; Christiansen, Peter Leth; Lomdahl, P. S.}

\section{Published in:}

Acoustical Society of America. Journal

Link to article, DOI:

10.1121/1.391312

Publication date:

1984

\section{Document Version}

Publisher's PDF, also known as Version of record

Link back to DTU Orbit

Citation $(A P A)$ :

Sørensen, M. P., Christiansen, P. L., \& Lomdahl, P. S. (1984). Solitary waves on nonlinear elastic rods. I. Acoustical Society of America. Journal, 76(3), 871-879. https://doi.org/10.1121/1.391312

\section{General rights}

Copyright and moral rights for the publications made accessible in the public portal are retained by the authors and/or other copyright owners and it is a condition of accessing publications that users recognise and abide by the legal requirements associated with these rights.

- Users may download and print one copy of any publication from the public portal for the purpose of private study or research.

- You may not further distribute the material or use it for any profit-making activity or commercial gain

- You may freely distribute the URL identifying the publication in the public portal

If you believe that this document breaches copyright please contact us providing details, and we will remove access to the work immediately and investigate your claim 


\title{
Solitary waves on nonlinear elastic rods. I
}

\author{
M. P. Soerensen, P. L. Christiansen, and P. S. Lomdahla) \\ Laboratory of Applied Mathematical Physics, The Technical University of Denmark, DK-2800 Lyngby, \\ Denmark
}

(Received 22 September 1982; accepted for publication 26 April 1984)

\begin{abstract}
Acoustic waves on elastic rods with circular cross section are governed by improved Boussinesq equations when transverse motion and nonlinearity in the elastic medium are taken into account. Solitary wave solutions to these equations have been found. The present paper treats the interaction between the solitary waves numerically. It is demonstrated that the waves behave almost like solitons in agreement with the fact that the improved Boussinesq equations are nearly integrable. Thus three conservation theorems can be derived from the equations. A new subsonic quasibreather is found in the case of a cubic nonlinearity. The balance between dispersion and nonlinearity in the equation is investigated.
\end{abstract}

PACS numbers: 43.25.Cb, 43.40.Cw

\section{INTRODUCTION}

In recent years exact soliton solutions to approximately 50 nonlinear partial differential equations (PDEs) or systems of PDEs have been found. Solitons are solitary waves, i.e., localized, nonsingular waves (like humps or kinks) which can propagate without changing their shape. In addition solitons retain their form after mutual interaction. The existence of such stable solutions is due to a balance between dispersion and nonlinearity in the PDEs. Soliton solutions are also linked to the exact integrability of the PDEs, thus to the existence of infinitely many conservation theorems for the equations. The first conservation theorems may be interpreted physically, for example, as conservation of energy and momentum. The methods by which the exact soliton solutions have been obtained are essentially the Bäcklund transformation, Hirota's method of bilinear differential operators, and the inverse scattering transform. Nearly all known solitons are solutions to evolution equations in one spatial dimension. Among a number of recent monographs of the subject we mention the textbook by G. L. Lamb ${ }^{1}$ and the survey edited by R. K. Bullough and P. J. Caudrey. ${ }^{2}$

Most physical systems are modeled by nonlinear PDEs which are not exactly integrable. However, some of the equations are what we shall denote nearly integrable. At present, we can only offer an approximate definition of this class of equations. First, the equation must possess solitary wave solutions as well as a finite number of conservation theorems. Second, the PDE must reduce to an exactly integrable equation in the small amplitude limit. Finally, a numerical treatment of the interaction between two solitary waves must show that the waves essentially retain their shape after the interaction. Thus only a small amount of linear disturbance may be created as a result of the interaction.

In the present paper the propagation of acoustical waves on a circular elastic rod is investigated. Nariboli $^{3}$ first presented a detailed analysis of the boundary value problem for the cylindrical rod corresponding to vanishing surface stresses based on a consistent perturbation procedure. He uses a polynomial form of the elastic energy in the quadratic approximation and justifies the validity in the "small" am-

\footnotetext{
a) Present address: Center for Nonlinear Studies, Los Alamos National Laboratory, University of California, Los Alamos, NM 87545.
}

plitude case. Physical nonlinearity due to a nonlinear constitutive relation for the elastic medium is thus neglected, while the geometrical nonlinearity resulting from the Lagrangian strain tensor is treated rigorously. A PDE for the longitudinal strain which is closely related to the Korteweg-de Vries equation is obtained. An explicit single solitary wave solution is given. Ostrovskii and Sutin include physical nonlinearity by starting with the Taylor expansion of the elastic energy with respect to the Lagrangian strain tensor. Their treatment, which neglects geometrical nonlinearity, leads to a fourth-order PDE related to Boussinesq's equation (BE). The BE contains $u_{x x x x}$ and $\left(u^{2}\right)_{x x}$ and is exactly integrable. ${ }^{5}$ In the PDE used by Ostrovskii and Sutin the term $u_{x x u t}$, which accounts for the transverse motion, replaces $u_{x x x x}$ and destroys the exact integrability. Iskander and Jain ${ }^{6}$ denoted this equation the improved Boussinesq equation (IBE) and investigated numerically head-on collision between solitary waves of the type found by Bogolubsky. ${ }^{7}$ The authors of Ref. 6 computed the breakup of an initially static wave. They concluded that the solitary waves interacted almost elastically (i.e., like solitons) for smaller amplitudes of the waves. For increasing amplitudes they found a gradual transition to inelastic behavior.

Bogolubsky ${ }^{7}$ considered a modified version of the IBE in which $\left(u^{2}\right)_{x x}$ is replaced by $\left(u^{3}\right)_{x x}$. This equation may be denoted the modified improved Boussinesq equation (MIBE). A solitary wave solution to this equation was found in Ref. 7. The same nonlinearity $\left[\left(u^{3}\right)_{x x}\right]$ has recently been considered in a study of seismological waves by Bataille and Lund $^{8}$ in which a Boussinesq equation modified in this manner occurs.

Equations like BE, IBE, and MIBE also occur in the continuum limit description of nonlinear lattices, in plasma physics, and nonlinear electric transmission lines. In the latter example position $x$ and time $t$ may be interchanged in the equations. ${ }^{9.10}$ Present literature contains an increasing number of investigations of nearly integrable systems.

The present paper is structured in the following manner: in Sec. I we derive the fourth-order PDE for the spatial derivative of the displacement along the rod from the Lagrangian for systems which include nonlinear terms up to fourth order in the elastic energy. ${ }^{11}$ The reader may consult 
the references listed in the work ${ }^{12}$ for the experimental determination of the corresponding elastic coefficients. Particular cases considered are the third-order case and the genuine fourth-order case with arbitrary signs of the elasticity coefficients. For the homogeneous rods the resulting PDEs for the strain reduce to IBE and MIBE, respectively. Conservation theorems for energy, momentum, and total displacement are found.

Section II presents the solitary wave solutions to IBE and MIBE and discusses the influence of the nonlinear term and the dispersive term on the solutions. The values of the conserved quantities for the solitary waves are given.

In Sec. III IBE and MIBE are reduced to the Korteweg-de Vries equation (KdV) and the modified Korteweg-de Vries equation (mKdV), respectively, by means of the reductive perturbation technique ${ }^{13}$ in the small amplitude limit. This procedure thus lowers the order of the PDE from four to three. As a consequence, propagation in only one spatial direction is permitted. In IBE and $\mathrm{KdV}$ the nonlinearity is an even function of the solution in contrast to MIBE and $\mathrm{mKdV}$ where the nonlinearity is odd. As a consequence only soliton solutions are found in the former case while both solitons and antisolitons exist in the latter case. Solitions and antisolitons may be combined into a lower energy state called a breather. The analytical expression for the $\mathrm{mKdV}$ breather is listed because it serves as initial condition in our numerical solution of MIBE in Sec. IV. In this section our numerical solution of initial value problems for IBE and MIBE is presented. We shall consider propagation; collision, and breakdown of solitary waves and a new quasibreather solution for MIBE. In two appendices we treat the elastic coefficients and the numerical schemes used for the computations.

A sequel to the present paper will treat reflection of solitary waves at an end of the elastic rod with realistic boundary conditions. Also reflection and transmission of solitary waves at continuous and discontinuous changes in the cross-section area of the elastic rod are investigated. Fission of solitary waves is observed.

\section{DERIVATION OF WAVE EQUATIONS}

We define the longitudinal displacement component of a plane cross section along the isotropic circular-cylindrical $\operatorname{rod}$ as $u_{1} \equiv W(X, T)$ where $X$ is the position of the undisturbed cross section and $T$ is time. The exact solution for the linear elastic (Hookeian) rod ${ }^{13-18}$ shows that $u_{1}$ is independent of the radial variable $R=\left(Y^{2}+Z^{2}\right)^{1 / 2}$ in the limit of long wavelength. Similarly, $u_{1}$ is assumed to be independent of $R$ for a thin nonlinear elastic rod as long as the width of the solitary wave solution is large compared with the diameter of the rod.

For an isotropic cylindrical rod under uniform longitudinal tension the deformation is homogeneous and the radial displacement, $u_{\mathrm{rad}}$, is exactly given by ${ }^{19}$

$$
u_{\text {rad }}=U(X, R)=R F\left(W_{X}\right) \text {, }
$$

where $W_{X}=\partial W / \partial X$ and $F$ is a nonlinear function. If $F\left(W_{X}\right)$ may be expanded in the form of a Taylor series,

$$
F\left(W_{X}\right)=-\sigma_{1} W_{X}-\sigma_{2} W_{X}^{2}-\sigma_{3} W_{X}^{3}-\cdots,
$$

the expansion coefficients are the first- and higher-order Poisson ratios for which expressions in terms of the secondand higher-order elastic constants are available up to fourth order. ${ }^{20.21}$ For longitudinal elastic waves in an isotropic linear elastic rod the radial displacement in the long wavelength limit is given by ${ }^{13-18} u_{\mathrm{rad}}=-\sigma R W_{X}$. Equation (1) is assumed to be valid also in the time-dependent inhomogeneous nonlinear case where the width of the solitary wave is large compared with radius of the rod

$$
u_{\mathrm{rad}}=U(X, R, T)=R F\left[W_{X}(X, T)\right] .
$$

In the absence of torsional deformations the displacement gradient, $\partial u_{i} / \partial X_{j}$, corresponding to the displacement field $u_{1}=W(X, T), u_{\mathrm{rad}}=U(X, R, T)$ given by Eq. (3) becomes

$$
\frac{\partial u_{i}}{\partial X_{R}}=\left\{\begin{array}{lll}
W_{X} & 0 & 0 \\
Y F^{\prime} W_{X X} & F & 0 \\
Z F^{\prime} W_{X X} & 0 & F
\end{array}\right\}
$$

in the Cartesian reference frame, $\left(X_{1}, X_{2}, X_{3}\right)=(X, Y, Z)$, in the material description. Here $F^{\prime}$ is

$$
F^{\prime}=\frac{d F}{d W_{X}}=-\sigma_{1}-2 \sigma_{2} W_{X}-3 \sigma_{3} W_{X}^{2}-\cdots
$$

according to Eq. (2).

Harmonic wave solutions in a linear elastic rod are given by $W(X, T)=A \exp [i(k X-\omega T)]$, where $A$ is the amplitude, $k$ is the wavenumber, and $\omega$ is the cyclic frequency. For these waves $\left|R W_{X X}\right|=k R\left|W_{X}\right|$. Thus in the long wavelength limit $\left|R W_{X X}\right|<\left|W_{X}\right|$ since $k R<1$. The former condition is expected to hold in the nonlinear case when the width of the solitary wave is large compared with the radius of the rod. In conjunction with Eq. (5) for $\sigma_{n}<1(n=1,2, \ldots)$ and $\left|W_{x}\right|<1$ it follows that the off-diagonal elements in the displacement gradient may be neglected, and Eq. (4) becomes

$$
\frac{\partial u_{i}}{\partial X_{n}}=\left\{\begin{array}{ccc}
W_{X} & 0 & 0 \\
0 & F & 0 \\
0 & 0 & F
\end{array}\right\}
$$

with $F$ given by Eq. (2).

With the displacement gradient given by Eq. (6) and $F$ given by Eq. (2) the elastic energy density (see Fig. 1) becomes

$$
\varphi=\frac{1}{2 !} E_{2} W_{X}^{2}+\frac{1}{3 !} E_{3} W_{X}^{3}+\frac{1}{4 !} E_{4} W_{X}^{4}
$$

to the fourth order. Here $E_{2}$, Young's modulus, and the higher order expansion coefficients, $E_{3}$ and $E_{4}$, are given in Appendix A. The potential energy density is

$$
\mathscr{V}=S \varphi,
$$

where $S$ is the cross-sectional area.

The corresponding kinetic energy density (per length unit of the rod) is given by

$$
\mathscr{T}=\int_{S} \frac{1}{2} \rho\left(W_{T}^{2}+U_{T}^{2}\right) d S,
$$

where $\rho$ is the density of the elastic material. According to Eqs. (3) and (5) we get

$$
U_{T}=-R\left(\sigma_{1}+2 \sigma_{2} W_{X}+3 \sigma_{3} W_{X}^{2}+\cdots\right) W_{X T} .
$$

Assuming that the higher order terms in $\mathscr{T}$ resulting from Eq. (10) are small compared with the higher order terms in 

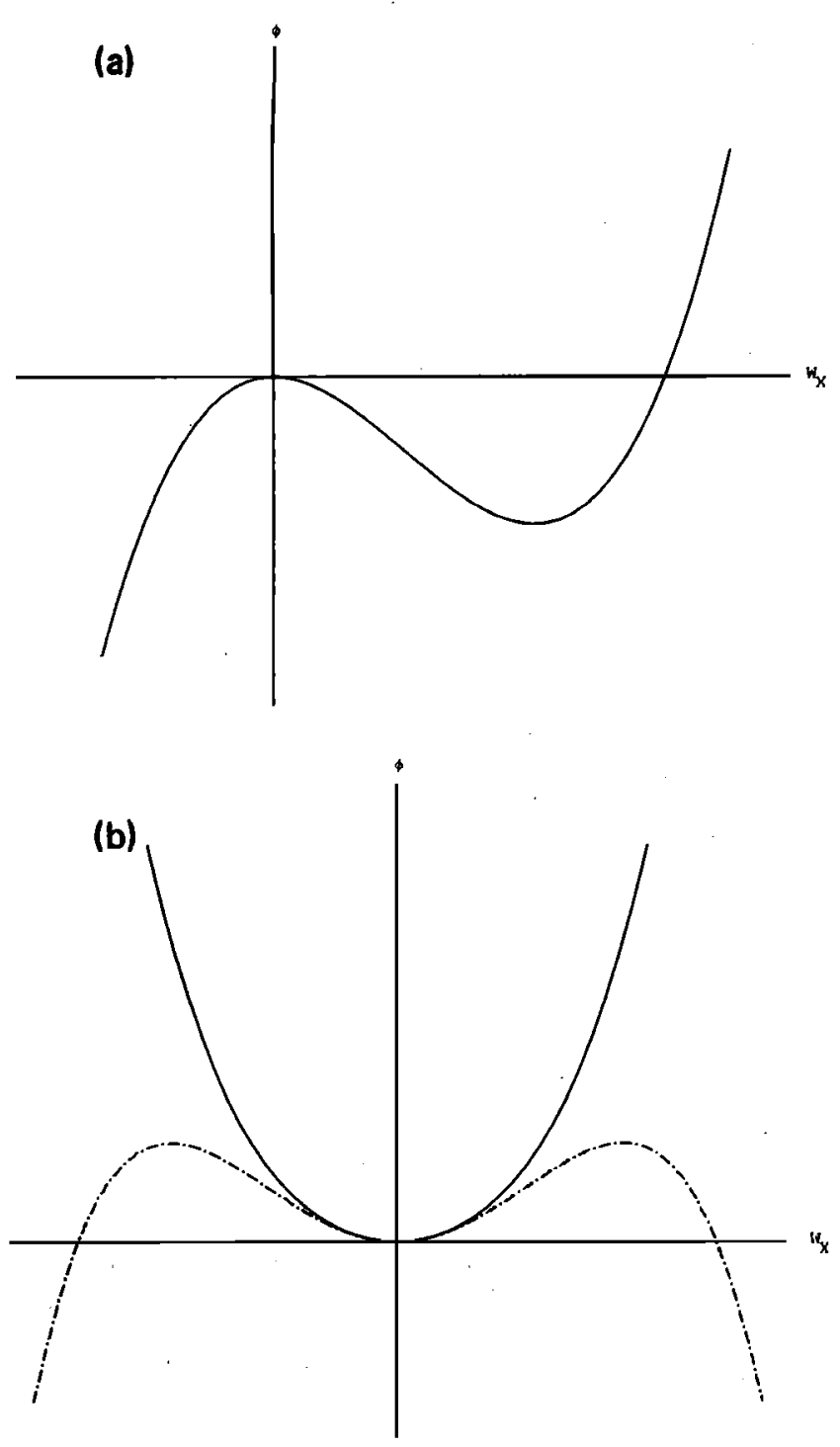

FIG. 1. Elastic energy $\phi$, as function of derivative of displacement, $W_{X}$. (a) Quadratic Hooke's law corresponding to $E_{3} \neq 0$ and $E_{4}=0$ in (3). (b) Cubic Hooke's law corresponding to $E_{3}=0$ and $E_{4}>0$ full curve (hard spring) and $E_{4}<0$ dot-and-dash line (soft spring) in (3).

Eq. (8) we get the Lagrangian density for the system

$$
\begin{aligned}
\mathscr{L}= & \mathscr{T}-\mathscr{V}=\frac{1}{2} S \rho\left[W_{T}^{2}+\left(S \sigma^{2} / 2 \pi\right) W_{X T}^{2}\right] \\
& -\frac{1}{2} S E_{2} W_{X}^{2}-\frac{1}{6} S E_{3} W_{X}^{3}-\frac{1}{24} S E_{4} W_{X}^{4} .
\end{aligned}
$$

The coefficients $E_{2}, E_{3}, E_{4}, \sigma, S$, and $\rho$ may depend on $X$. The Euler equation for this Lagrangian density is

$$
\frac{\partial}{\partial X} \frac{\partial \mathscr{L}}{\partial W_{X}}+\frac{\partial}{\partial T} \frac{\partial \mathscr{L}}{\partial W_{T}}-\frac{\partial^{2}}{\partial X \partial T} \frac{\partial^{2} \mathscr{L}}{\partial W_{X T}}=0
$$

yielding

$$
\begin{array}{r}
\frac{\partial}{\partial X}\left(S E W_{X}\right)+\frac{1}{2} \frac{\partial}{\partial X}\left(S E_{3} W_{X}^{2}\right)+\frac{1}{3} \frac{\partial}{\partial X}\left(S E_{4} W_{X}^{3}\right) \\
-\frac{\partial}{\partial T}\left(S \rho W_{T}\right)+\frac{\partial^{2}}{\partial X \partial T}\left(\frac{S^{2} \rho \sigma^{2}}{2 \pi} W_{X T}\right)=0 .
\end{array}
$$

From the Hamiltonian density, $\mathscr{H}=\mathscr{T}+\mathscr{V}$, we get the Hamiltonian

$$
\boldsymbol{H}=\int_{-\infty}^{\infty}(\mathscr{T}+\mathscr{V}) d X
$$

which is a conserved quantity since $d H / d T=0$ when $W$ satisfies (13). Another conserved quantity is the momentum defined as

$$
P=\int_{-\infty}^{\infty} S \rho W_{T} d X
$$

[Integration of (9) with respect to $X$ yields $d P / d T=0$ when zero boundary conditions for $W_{X}$, etc. at infinity are applied.]

$$
\begin{aligned}
& \text { An equivalent formulation of }(13) \text { is } \\
& \frac{\partial \Omega}{\partial X}=W_{X T}-\frac{\partial}{\partial X}\left\{\frac{1}{S \rho}\left[\frac{\partial}{\partial X}\left(\frac{S^{2} \rho \sigma^{2}}{2 \pi} W_{X T}\right)\right]\right\}
\end{aligned}
$$

and

$$
\frac{\partial \Omega}{\partial T}=\frac{1}{S \rho} \frac{\partial}{\partial X}\left[S\left(E W_{X}+\frac{1}{2} E_{3} W_{X}^{2}+\frac{1}{3} E_{4} W_{X}^{3}\right)\right],
$$

where $\Omega=T(X, T)$. Integration of (16a) with respect to $X$ yields $d D / d T=0$ where the total displacement $D$ is given by

$$
D=\int_{-\infty}^{\infty} W_{X} d X
$$

when zero boundary conditions for $\Omega$ and $W_{X}$ at infinity are used.

For a homogeneous rod (13) reduces to

$$
w_{x x}-w_{t s}+f\left(w_{x}\right) w_{x x}+w_{x x t t}=0
$$

in dimensionless coordinates $x=X /(\sigma \sqrt{S / 2 \pi})$ and

$$
t=T \sqrt{E_{2}} /(\sigma \sqrt{S \rho / 2 \pi})
$$

in the following special cases: for $E_{3} \neq 0$ and $E_{4}=0$ (material with quadratic Hooke's law) we introduce

$$
w(x, t)=W(X, T) E_{3} /\left(E_{2} \sigma \sqrt{S / 2 \pi}\right) .
$$

As a result $f\left(w_{x}\right)=w_{x}$. For $E_{3}=0$ and $E_{4} \gtrless 0$ (material with cubic Hooke's law) we introduce

$$
w(x, t)=W(X, T) \sqrt{\left|E_{4}\right|} /\left(\sigma \sqrt{S E_{2} / 2 \pi}\right)
$$

and $f\left(w_{x}\right)= \pm w_{x}^{2}$.

The dimensionless strain $u=w_{x}$, satisfies (18) with the nonlinear term replaced by $\frac{1}{2}\left(u^{2}\right)_{x x}$ and $\pm \frac{1}{3}\left(u^{3}\right)_{x x}$ in the quadratic and cubic cases (B1). These equations are denoted IBE and MIBE, respectively.

\section{SOLITARY WAVE SOLUTIONS AND CONSERVED QUANTITIES}

In the quadratic case a solitary strain wave is $u(x, t)=3\left(c^{2}-1\right) \operatorname{sech}^{2}\left[\left(\sqrt{c^{2}-1} / 2 c\right)\left(x-x_{0}-c t\right)\right]$,

where the velocity $c$ satisfies $|c|>1$ and $x=x_{0}$ is the position of the wave at $t=0$. [For $|c|<0 \mathrm{Eq}$. (19) yields an unbounded negative periodic solution. For $c= \pm 1$ we get the negative unbounded localized solution $u=-12 /\left(x-x_{0} \mp t\right)^{2}$.]

In the cubic case with the nonlinear term $+\frac{1}{3}\left(u^{3}\right)_{x x}$ we find solitary and antisolitary strain waves

$u(x, t)= \pm \sqrt{6} \sqrt{c^{2}-1} \operatorname{sech}\left[\left(\sqrt{c^{2}-1} / c\right)\left(x-x_{0}-c t\right)\right]$

for upper and lower sign and $|c|>1$. (Real solutions for $|c|<1$ are not found.) In the cubic case with nonlinear term 
$-\frac{1}{3}\left(u^{3}\right)_{x x}$ we get unbounded localized solutions for $|c| \geqslant 1$ and unbounded periodic solutions for $|c|<1$. Equation (20) shows that both solitary and antisolitary waves exist in the cubic case with +$\}\left(u^{3}\right)_{x x}$ term in contrast to the quadratic case where only positive solitary waves are found.

In the absence of the dispersive term, $u_{x x m}$, in the wave equations we get the nonlinear simple wave solution $u=f[x-c(u) t]$ where $f$ is an arbitrary function and $c(u)=\sqrt{1+u}$ or $\sqrt{1 \pm u^{2}}$ in the quadratic and cubic cases $\pm f\left(u^{3}\right)_{x x}$. Thus the amplitude dispersion makes the larger amplitudes $|u|$, move faster or slower than the sinaller amplitudes causing a steepening of the front or the back side of the wave. We shall see an example of this effect in Sec. IV.

If, on the other hand, the nonlinear term is cancelled in the wave equation we get the linear solution

$$
u=\int d k \tilde{u}(k) \exp \left[i k\left(x-t / \sqrt{1+k^{2}}\right)\right],
$$

where $\tilde{u}(k)$ is the spectrum of $u$ as function of the wavenumber $k$. The group velocity for this wave is $c_{g}=\left(1+k^{2}\right)^{-3 / 2}$. Thus the frequency dispersion for this wave makes the components with larger wavenumbers move slower. This tendency may balance steepening of the wave due to the nonlinearity. As a result we find a positive solitary wave in the $\frac{1}{2}\left(u^{2}\right)_{x x}$ case and positive and negative solitary waves in the $y_{3}\left(u^{3}\right)_{x x}$ case. As we shall see in Sec. IV the two mechanisms do not balance each other for a negative wave solution in the quadratic case and in the $-\frac{1}{3}\left(u^{3}\right)_{x x}$ case.

From (14), (15), and (17) we derive the conserved quantities for the solution (19)

$$
\begin{aligned}
& H=\left(E_{2}^{3} \sigma S \sqrt{S / 2 \pi} / E_{3}^{2}\right) h \\
& h=24 c^{2}|c|\left(c^{2}-1\right)^{3 / 2} \\
& P=\left[\left(E_{2} \rho S\right)^{3 / 2} / E_{3} \sqrt{2 \pi}\right] p, \\
& P=-c d
\end{aligned}
$$

and

$$
\begin{aligned}
& D=\left(E_{2} \sigma \sqrt{S / 2 \pi} / E_{3}\right) d, \\
& d=12|c|\left(c^{2}-1\right)^{1 / 2}
\end{aligned}
$$

with $|c|>1$. Here we have introduced the corresponding normalized quantities $h, p$, and $d$, which are conserved in the case of a homogeneous rod.

The conserved quantities for the solution (20) become

$$
\begin{aligned}
& H=\left(E_{2}^{2} \sigma S \sqrt{S / 2 \pi} /\left|E_{4}\right|\right) h, \\
& h=12 c^{2}|c|\left(c^{2}-1\right)^{1 / 2} ; \\
& P=\left(E_{2} \sigma S \sqrt{\rho S / 2 \pi} / \sqrt{\left|E_{4}\right|}\right) p, \quad p=-c d ;
\end{aligned}
$$

and

$$
D=\left(\sigma \sqrt{S E_{2} / 2 \pi} / \sqrt{\left|E_{4}\right|}\right) d, \quad d= \pm \pi \sqrt{6}|c| ;
$$

with $|c|>1$.

The relationship $p=-c d$ in both cases can be seen directly from the traveling wave assumptions (19) and (20). Note that $|d|$ and $|p|$ have the lower bound $\pi \sqrt{6}$ in the cubic case.

\section{RELATIONSHIP TO INTEGRABLE EQUATIONS}

In order to illustrate the relationship of our wave equations to integrable equations we use the reductive perturbation expansion ${ }^{22}$

$$
u(x, t)=\sum_{n=1}^{\infty} \epsilon^{n} u_{n}(\xi, r), \quad 0<\epsilon<1,
$$

with

$$
\xi=\epsilon[(x-t) / \sqrt{6}], \quad \tau=\epsilon^{3}(x / 12 \sqrt{6}),
$$

and

$$
\xi=\epsilon^{1 / 2}[(x-t) / \sqrt{6}], \tau=\epsilon^{3 / 2}(x / 12 \sqrt{6}),
$$

respectively. To the lowest order we then obtain the KdV equation

$$
u_{1 \tau}+6 u_{1} u_{1 \xi}+u_{1 \xi \xi \xi}=0
$$

from IBE and the $\mathrm{mKdV}$ equations

$$
u_{1 \tau} \pm 6 u_{1}^{2} u_{1 \xi}+u_{1 \xi \xi \xi}=0
$$

from MIBE.

The KdV equation and the mKdV equation with the upper sign are integrable and multisoliton solutions have been found. In the latter case antisoliton solutions exist and soliton and antisoliton may combine into a breather. ${ }^{23}$ The resulting small amplitude solution becomes

$$
\begin{aligned}
u(x, t)= & -4 \epsilon \beta \frac{\cos \Phi-(\beta / \alpha) \sin \Phi \tanh \Psi}{1+(\beta / \alpha)^{2} \sin \Phi \operatorname{sech}^{2} \Psi} \operatorname{sech} \Psi \\
& +O\left(\epsilon^{2}\right)
\end{aligned}
$$

with

$$
\begin{aligned}
& \Phi=(2 \epsilon \alpha / \sqrt{6})\left\{\left[1+\left(\frac{1}{3} \alpha^{2}-\beta^{2}\right) \epsilon^{2}\right] x-t\right\}, \\
& \Psi=(2 \epsilon \beta / \sqrt{6})\left\{\left[1+\left(\alpha^{2}-1 \beta^{2}\right) \epsilon^{2}\right] x-t\right\},
\end{aligned}
$$

$\alpha$ and $\beta$ being positive constants. The resulting group and phase velocities for the breather are $c_{\mathrm{g}} \cong 1+\left(\beta^{2} / 3-\alpha^{2}\right) \epsilon^{2}$ and $c_{\mathrm{ph}} \simeq 1+\left(\beta^{2}-\alpha^{2} / 3\right) \epsilon^{2}$, respectively. (An example is shown in Fig. 10.)

We note that the $m K d V$ equation (31) with the lower sign has a shock wave type solution. ${ }^{24}$

\section{NUMERICAL RESULTS}

In this section we present our numerical solutions of the following initial value problems

$$
\text { or } \left.\begin{array}{cc}
u_{x x}-u_{t t}+\frac{1}{2}\left(u^{2}\right)_{x x}+u_{x x t t}=0 \\
u_{x x}-u_{t t}+\frac{1}{3}\left(u^{3}\right)_{x x}+u_{x x t t}=0
\end{array}\right\} \begin{aligned}
& 0<x<l, \\
& 0<t<t_{f},
\end{aligned}
$$

and

$$
\begin{aligned}
& u(0, t)=u(l, t)=0, \\
& u(x, 0)=f(x, 0) \text { and } u_{t}(x, 0)=f_{t}(x, 0) .
\end{aligned}
$$

In the initial condition (35) $f(x, t)$ will be specified for each numerical experiment. The results are obtained by the finite difference scheme described in Appendix B.

In Figs. 2 and 3 we show that solitons are able to survive head-on collision in the case of a quadratic nonlinearity (33a) and a cubic nonlinearity (33b). In the initial conditions (35) we use 


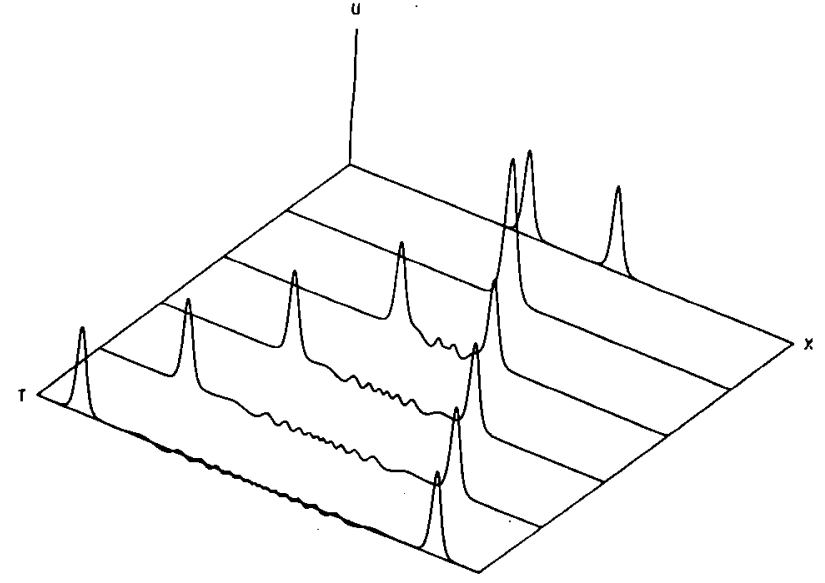

FIG. 2. Head-on collision between two solitary waves in quadratic case. Numerical solution of (33a) $(36 \mathrm{a})$ with $l=200, t_{f}=66, N=2$, $c_{1}=-c_{2}=1.4, x_{01}=80$, and $x_{02}=120$.

$f(x, t)=\sum_{i=1}^{N} 3\left(c_{i}^{2}-1\right) \operatorname{sech}^{2}\left(\frac{\sqrt{c_{i}^{2}-1}}{2 c_{i}}\left(x-x_{0 i}-c_{i} t\right)\right)$

and

$f(x, t)=\sum_{i=1}^{N} \delta_{i} \sqrt{6} \sqrt{c_{i}^{2}-1} \operatorname{sech}\left(\frac{\sqrt{c_{i}^{2}-1}}{c_{i}}\left(x-x_{0 i}-c_{i} t\right)\right)$

respectively. Here $N$ is the number of solitary waves. The solitons retain their shape and velocity after the collision in both cases. The radiation field between the solitons after the collision is slightly stronger in the cubic case than in the quadratic case. Similarly, Figs. 4 and 5 show takeover in the two cases. Even though the maximal value of $u$ is approximately three times as big in the quadratic case as in the cubic case the radiation appears to be of the same order of magnitude. The approximately solitonic behavior can be explained by reducing the equations to the $\mathrm{KdV}$ and the $\mathrm{mKdV}$ equations by means of the reductive perturbation theory as shown in Sec. III and invoking the properties of the soliton solutions of these equations. As we saw in Sec. II only positive solitary waves exist in the quadratic case. However, in the cubic case we have found solitary waves and antisolitary waves. Figures 6 and 7 illustrate head-on collision and ta-

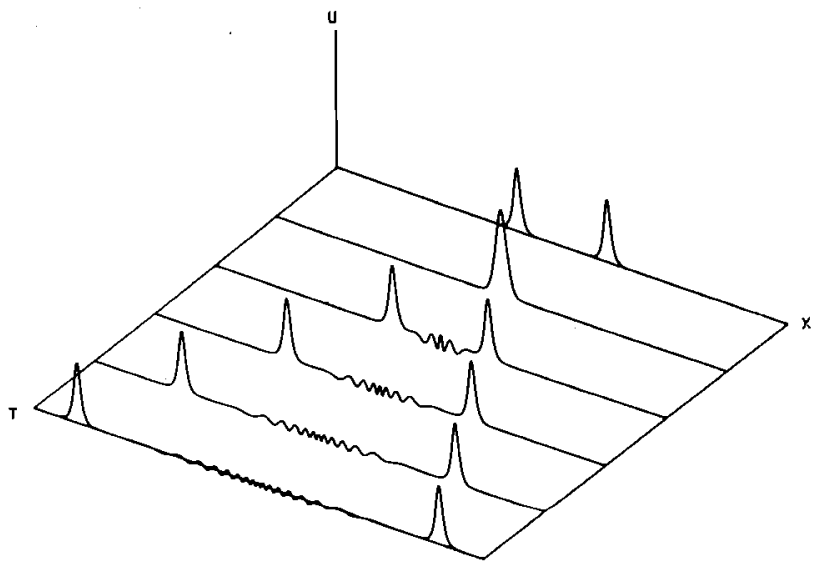

FIG. 3. Head-on collision between two solitary waves in cubic case. Numerical solution of $(33 \mathrm{~b})(36 \mathrm{~b})$ with $l=200, t_{f}=66, N=2, \delta_{1}=\delta_{2}=1$, $c_{1}=-c_{2}=1.5, x_{01}=80$, and $x_{02}=120$.

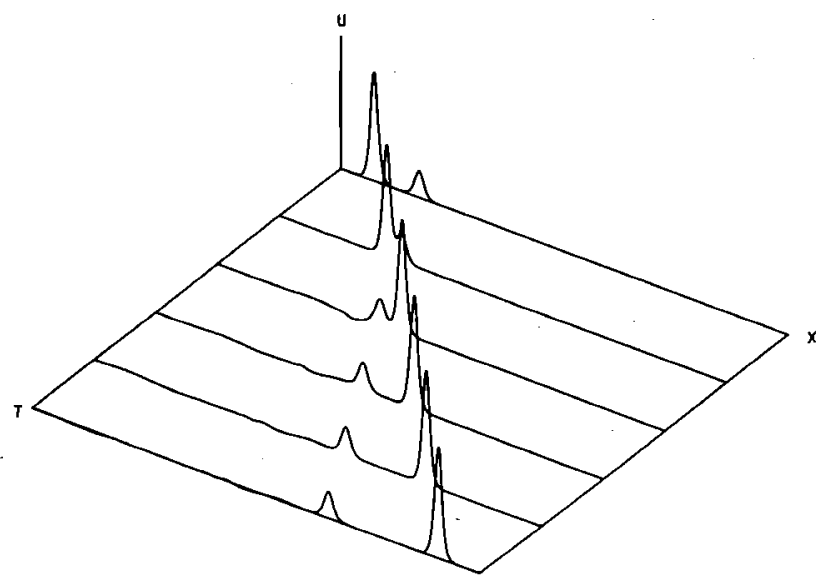

FIG. 4. Takeover of two solitary waves in the quadratic case. Numerical solution of (33a) $-(36 \mathrm{a})$ with $l=200, t_{f}=66, N=2, c_{1}=2.5, c_{2}=1.5$, $x_{01}=10$, and $x_{02}=20$.

keover of such waves. The radiation is stronger in both cases than for the corresponding solitary-solitary wave interaction. Figure 8 shows a solitary wave and an antisolitary wave traveling closely together with the same velocity in a breatherlike bound state. The total momentum of this wave is zero while each of its components has a momentum $p$, with absolute value $>\pi \sqrt{6}$. The wave travels with a decreasing group velocity $<1$. For $t>t_{f}$ we have observed that the wave stops and becomes singular. In Fig. 9 we have two antisolitary waves and one solitary wave. In this case one of the antisolitary waves and the solitary wave travel in a breatherlike state as in Fig. 8. The other antisolitary wave takes off with a velocity which is slightly smaller $(c=1.15)$ than the initial velocity $(c=1.3)$.

In Fig. 10 we consider the collision between two breathers of small amplitude. In our initial condition $f(x, t)$ is now the sum of two $\mathrm{mKdV}$ breathers traveling in opposite directions

$$
\begin{aligned}
& f(x, t) \\
& \quad=-4 \epsilon \sum_{i=1}^{2} \frac{\cos \Phi_{i}-\left(\beta_{i} / \alpha_{i}\right) \sin \Phi_{i} \tanh \Psi_{i}}{1+\left(\beta_{i} / \alpha_{i}\right)^{2} \sin \Phi_{i} \operatorname{sech}^{2} \Psi_{i}} \operatorname{sech} \Psi_{i},
\end{aligned}
$$

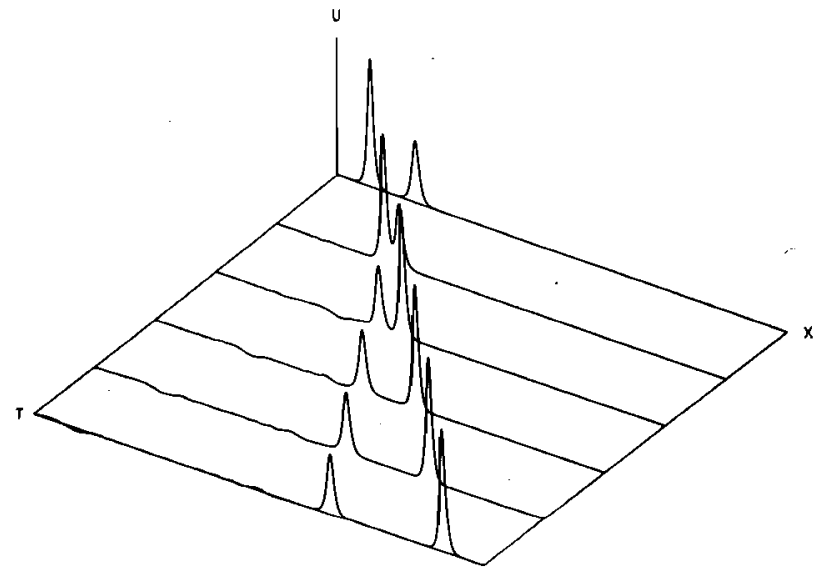

FIG. 5. Takeover of two solitary waves in the cubic case. Numerical solution of (33b) $-(36 \mathrm{~b})$ with $l=200, t_{f}=66, N=2, \delta_{1}=\delta_{2}=1, c_{1}=2.5$, $c_{2}=1.5, x_{01}=10$, and $x_{02}=20$. 


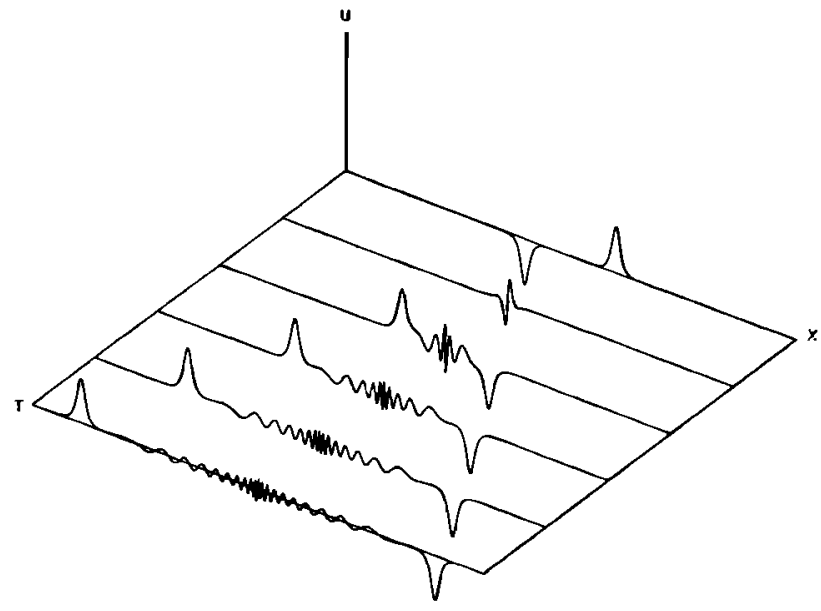

FIG. 6. Head-on collision between solitary and antisolitary wave in cubic case. Nurnerical solution of $(33 \mathrm{~b})(36 \mathrm{~b})$ with $I=200, t_{f}=77, N=2$, $-\delta_{1}=\delta_{2}=1, c_{1}=-c_{2}=1.3, x_{01}=80$, and $x_{02}=120$.

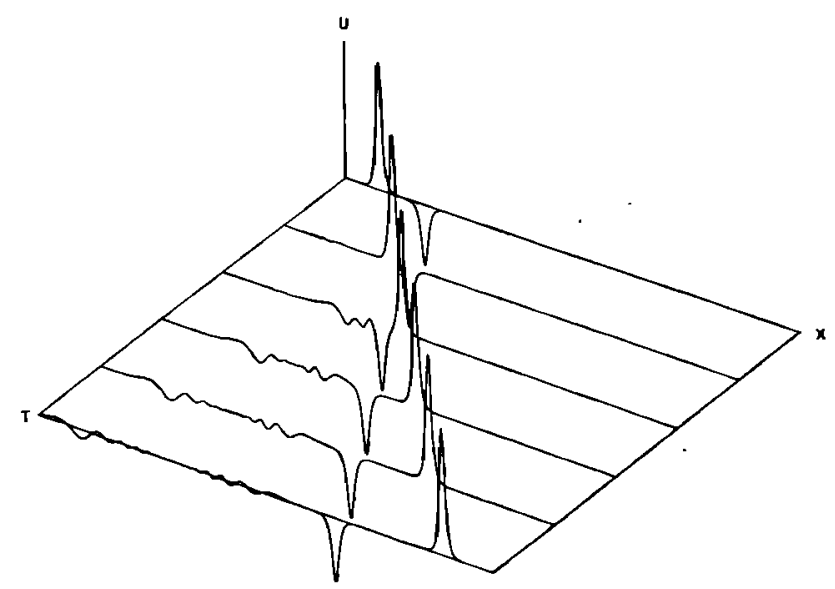

FIG. 7. Takeover of solitary and antisolitary wave in cubic case. Numerical solution of (33b) $(36 \mathrm{~b})$ with $I=200, t_{f}=66, N=2, \delta_{1}=-\delta_{2}=1$, $c_{1}=2.5, c_{2}=1.5, x_{01}=15$, and $x_{02}=35$.

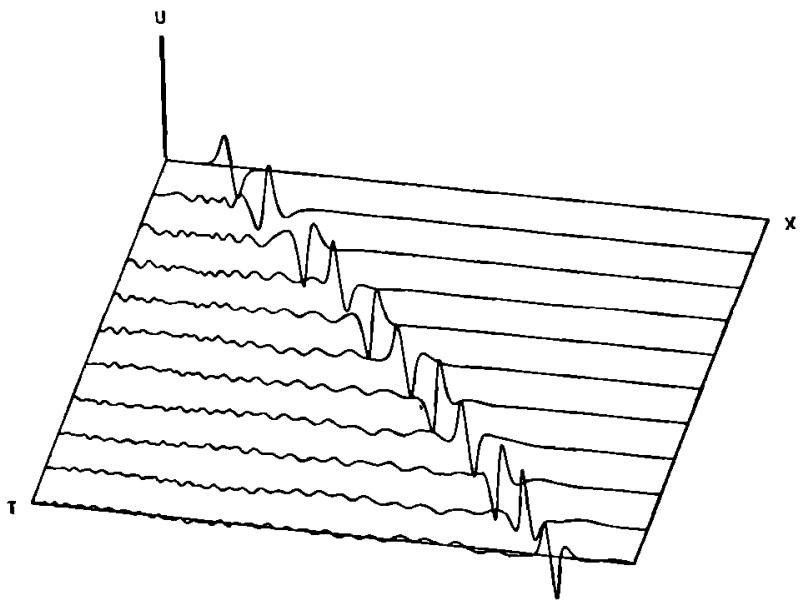

FIG. 8. Coupling of solitary and antisolitary wave in cubic case. Numerical solution of (33b) (36b) with $l=200, t_{f}=185, N=2, \delta_{1}=-\delta_{2}=1$, $c_{1}-c_{2}=1.3, x_{01}=20$, and $x_{02}=23$.

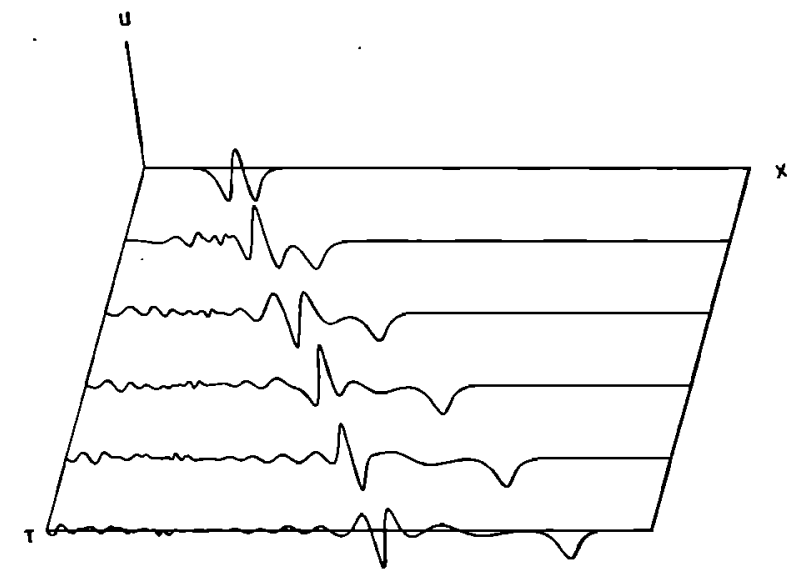

FIG. 9. Coupling of one solitary and two antisolitary waves in cubic case. Numerical solution of (33b) $(36 \mathrm{~b})$. Parameters $l=200, t_{f}=90, N=3$, $-\delta_{1}=\delta_{2}=-\delta_{3}=1, c_{1}=c_{2}=c_{3}=1.3, x_{01}=20, x_{02}=23$, and $x_{03}=26$.

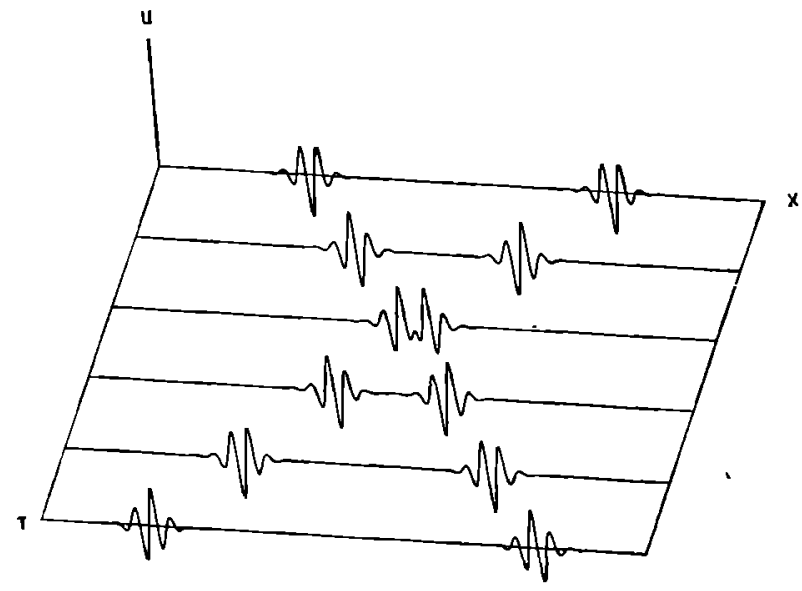

FIG. 10. Head-on collision between two small-amplitude breathers in cubic case. Numerical solution of (33b) $-(35)$ and (37). Parameters $l=400, t_{f}=50$, $\epsilon=0.1, \alpha_{1}=a_{2}=3, \beta_{1}=\beta_{2}=1, x_{01}=200$, and $x_{02}=600$.

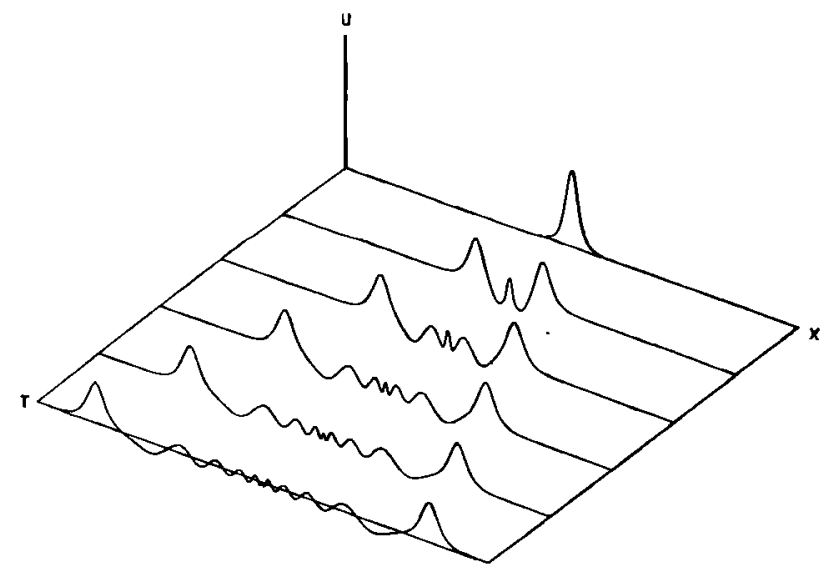

FIG. 11. Breakup of static wave in cubic case. Numerical solution of (33b)(35) and (38). Parameters $l=100, t_{f}=30, c=1.75$, and $x_{0}=50$. 
with

$$
\begin{aligned}
& \Phi_{i}=\frac{2 \epsilon \alpha_{i}}{\sqrt{6}}\left\{\left[1+\left(\frac{1}{3} \alpha_{i}^{2}-\beta_{l}^{2}\right) \epsilon^{2}\right]\left(x-x_{0 i}\right)+(-1)^{i} t\right\}, \\
& \Psi_{i}=\frac{2 \epsilon \beta_{i}}{\sqrt{6}}\left\{\left[1+\left(\alpha_{i}^{2}-\frac{1}{3} \beta_{i}^{2}\right) \epsilon^{2}\right]\left(x-x_{0}\right)+(-1)^{i} t\right\} .
\end{aligned}
$$

For the parameter values chosen the corresponding group and phase velocities for the two breathers become $c_{\mathrm{g}}= \pm 0.91$ and $c_{\mathrm{ph}}= \pm 0.98$. The breathers are seen to survive the collision with minimal radiation.

Figure 11 shows the breakup of a static wave given by

$$
f(x, t)=\sqrt{6} \sqrt{c^{2}-1} \operatorname{sech}\left[\left(\sqrt{c^{2}-1} / c\right)\left(x-x_{0}\right)\right] .
$$

This wave contains the potential energy $v=2|c|\left(2 c^{2}+1\right) \sqrt{c^{2}-1}$. For the parameters in the figure $v=35.8$. The static wave breaks up into two solitary waves with velocities $c= \pm 1.23$. According to (24) the corresponding energy is $2 \times 16.0=32.0$. Thus approximately $10 \%$ of the energy is lost to the radiation seen in the figure.

Figures 12 and 13 show the time evolution of two different negative solitary waves in the quadratic case. The waves are given by

$f(x, t)=-3\left(c^{2}-1\right) \operatorname{sech}^{2}\left[\left(\sqrt{c^{2}-1} / 2 c\right)\left(x-x_{0}-c t\right)\right]$.

In the former figure the velocity is so close to unity ( $c=1.1)$ that the amplitude (0.63) becomes so small that the dispersion term dominates. As a consequence the solitary waves evolve into an oscillating solution. In the latter figure $c=1.2$ and the amplitude becomes 1.32 . Now the solitary wave evolves into a singular solution very rapidly. Thus blowip has been shown to occur before $t=6$. Here the nonlinear term dominates and $c(u)=\sqrt{1+u}$ due to the amplitude dispersion. For the negative solitary wave the larger amplitudes ( < 1) will travel more slowly causing a steepening of the back front of the wave as seen in Fig. 13. For the initial condition (39) the amplitude value separating the oscillatory case and the blowup case has been determined to 0.83 . Similar results have been obtained for the $-\frac{1}{3}\left(u^{3}\right)_{x x}$ case. Here the initial condition (36b) with $n=1$ and $\delta_{1}=1$ yields the amplitude

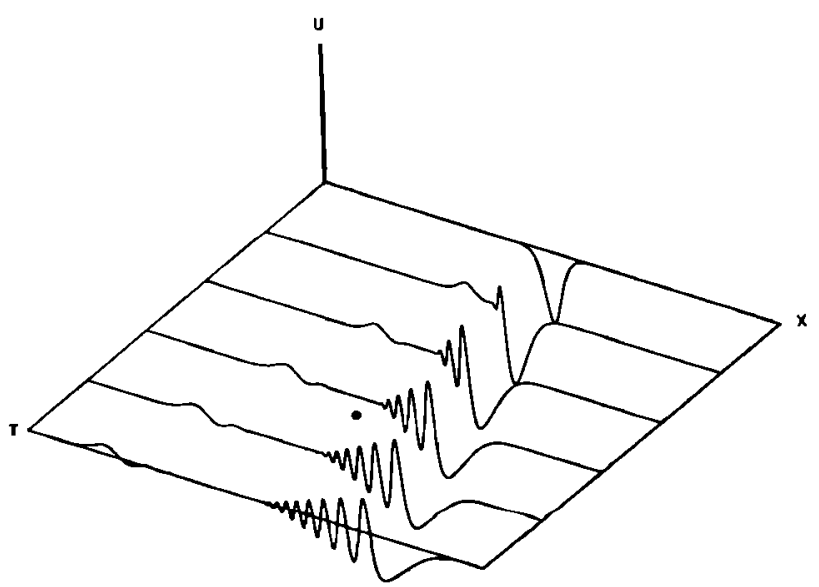

FIG. 12. Time evolution of negative solitary wave in quadratic case. Oscillatory solution results. Numerical solution of (33a) $-(35)$ and (39). Parameters $l=200, t_{f}=70, c=1.1$, and $x_{0}=100$.

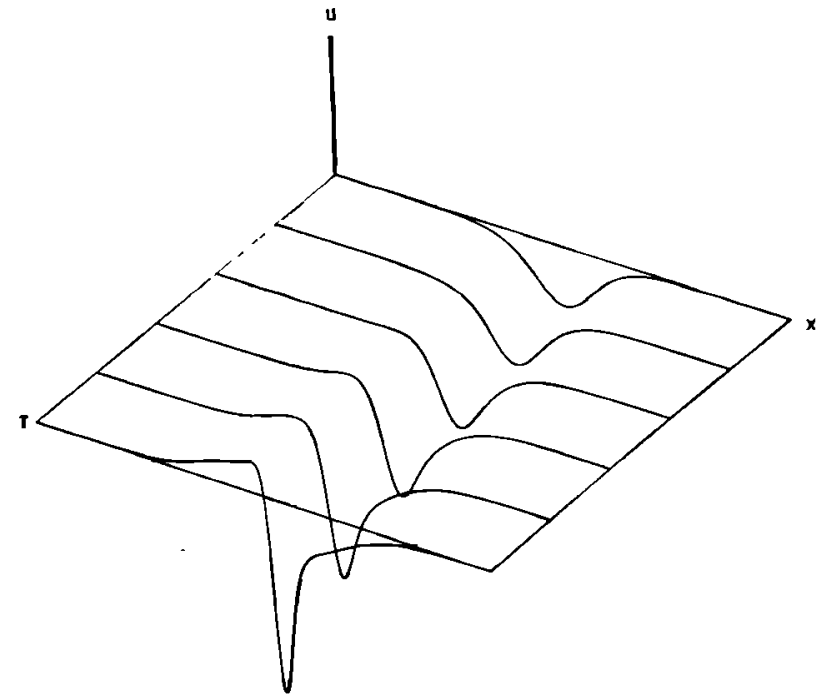

FIG. 13. Blowup of negative solitary wave in quadratic case. Numerical solution of (33a) $-(35)$ and (39). Parameters $l=40, t_{f}=5, c=1.2$, and $x_{0}=20$.

value 0.93 separating the oscillatory case and the blowup case.

\section{CONCLUSIONS}

Longitudinal waves on nonlinear elastic rods are governed by improved Boussinesq equations in the absence of torsional displacements provided that the length of the waves is large compared with the radial dimension of the rod.

Solitary wave solutions to the improved Boussinesq equations, modeling elastic rods with quadratic and cubic nonlinearities, have been investigated numerically. The waves turn out to behave almost like solitons, i.e., little linear radiation is created during their interaction. This result agrees with the fact that the improved Boussinesq equations in the present model are almost integrable, i.e., they reduce to the integrable $\mathrm{KdV}$ and $\mathrm{mKdV}$ equations in the small amplitude limit. Inclusion of neglected higher order terms may reduce the integrability of the system. A new quasibreather solution is found in the cubic case. Disturbance of the balance between dispersion and nonlinearity is shown to give oscillatory or singular behavior of the waves.

\section{ACKNOWLEDGMENTS}

The authors gratefully acknowledge the information on nonlinear elastic media we have received from $\mathbf{G}$. R. Barsch and J. Christoffersen. It is our pleasure to thank O. Skovgaard for many helpful discussions on the numerical methods.

\section{APPENDIX A}

The elastic energy up to fourth order can be written

$$
\begin{gathered}
\phi=\frac{1}{2} \lambda I_{1}^{2}+2 \mu I_{2}+A_{3} I_{1}^{3}+B_{3} I_{1} I_{2}+C_{3} I_{3} \\
+A_{4} I_{1}^{4}+B_{4} I_{1}^{2} I_{2}+C_{4} I_{2}^{2}+D_{4} I_{1} I_{3},
\end{gathered}
$$

where the three invariants are given by 


$$
I_{1}=\eta_{i i}, \quad I_{2}=\frac{1}{2} \eta_{i j} \eta_{j i}, \quad I_{3}=\frac{1}{3} \eta_{i j} \eta_{j k} \eta_{k i},
$$

where $\eta_{i j}$ is the Lagrangian strain tensor given by

$$
\eta_{i j}=\frac{1}{2} \frac{\partial u_{i}}{\partial X_{j}}+\frac{\partial u_{j}}{\partial X_{i}}+\frac{\partial u_{k}}{\partial X_{i}} \frac{\partial u_{k}}{\partial X_{j}},
$$

with summation over repeated indices. In Eq. (A1) $\lambda$ and $\mu$ are the Lamé constants, and $A_{3}, B_{3}, C_{3}$ and $A_{4}, B_{4}, C_{4}, D_{4}$ denote the third- and fourth-order isotropic elastic constants, respectively. Insertion of Eq. (6) into Eq. (A3) yields

$$
\eta_{i j}=\left(\begin{array}{ccc}
W_{X}+\frac{1}{2} W_{X}^{2} & 0 & 0 \\
0 & F+\frac{1}{2} F^{2} & 0 \\
0 & 0 & F+\frac{1}{2} F^{2}
\end{array}\right)
$$

and

$$
I_{1}=W_{X}+2 F+\frac{1}{2}\left(W_{X}^{2}+2 F^{2}\right),
$$

$$
\begin{aligned}
E_{2}= & \lambda\left(1-2 \sigma_{1}\right)^{2}+2 \mu\left(1+2 \sigma_{1}^{2}\right), \\
E_{3}= & 3 \lambda\left(1-2 \sigma_{1}\right)\left(1+2 \sigma_{1}^{2}-4 \sigma_{2}\right)+6 \mu\left(1+4 \sigma_{1} \sigma_{2}-2 \sigma_{1}^{3}\right) \\
& +6 A_{3}\left(1-2 \sigma_{1}\right)^{3}+3 B_{3}\left(1-2 \sigma_{1}\right)\left(1+2 \sigma_{1}^{2}\right)+2 C_{3}\left(1-2 \sigma_{1}^{3}\right)
\end{aligned}
$$

and

$$
\begin{aligned}
E_{4}= & 3 \lambda\left[\left(1+2 \sigma_{1}^{2}-4 \sigma_{2}\right)^{2}+16\left(1-2 \sigma_{1}\right)\left(\sigma_{1} \sigma_{2}-\sigma_{3}\right)\right]+6 \mu\left(1+2 \sigma_{1}^{4}+8 \sigma_{2}^{2}-24 \sigma_{1}^{2} \sigma_{2}+16 \sigma_{1} \sigma_{3}\right) \\
& +36 A_{3}\left(1-2 \sigma_{1}\right)^{2}\left(1+2 \sigma_{1}^{2}-4 \sigma_{2}\right)+6 B_{3}\left[2\left(1-2 \sigma_{1}\right)\left(1+4 \sigma_{1} \sigma_{2}-2 \sigma_{1}^{3}\right)\right. \\
& \left.+\left(1+2 \sigma_{1}^{2}-4 \sigma_{2}\right)\left(1+2 \sigma_{1}^{2}\right)\right]+12 C_{3}\left(1+2 \sigma_{1}^{4}-4 \sigma_{1}^{2} \sigma_{2}\right) \\
& +24 A_{4}\left(1-2 \sigma_{1}\right)^{4}+12 B_{4}\left(1-2 \sigma_{1}\right)^{2}\left(1+2 \sigma_{1}^{2}\right)+6 C_{4}\left(1+2 \sigma_{1}^{2}\right)^{2}+8 D_{4}\left(1-2 \sigma_{1}\right)\left(1-2 \sigma_{1}^{3}\right) .
\end{aligned}
$$

$$
\begin{aligned}
I_{2}= & \frac{1}{2}\left[W_{X}^{2}+2 F^{2}+W_{X}^{3}+2 F^{3}+\frac{1}{4}\left(W_{X}^{4}+2 F^{4}\right)\right], \\
I_{3}= & \frac{1}{3}\left[W_{X}^{3}+2 F^{3}+\frac{3}{2}\left(W_{X}^{4}+2 F^{4}\right)\right. \\
& \left.+\frac{3}{4}\left(W_{X}^{5}+2 F^{5}\right)+\frac{1}{8}\left(W_{X}^{6}+2 F^{6}\right)\right] .
\end{aligned}
$$

Substituting the expansion of $F$ in terms of $W_{X}$ from Eq. (2) we obtain

$$
\begin{aligned}
I_{1}= & \left(1-2 \sigma_{1}\right) W_{X}+\frac{1}{2}\left(1+2 \sigma_{1}^{2}-4 \sigma_{2}\right) W_{X}^{2} \\
& +2\left(\sigma_{1} \sigma_{2}-\sigma_{3}\right) W_{X}^{3}+O\left(W_{X}^{4}\right), \\
I_{2}= & \left(\frac{1}{2}+\sigma_{1}^{2}\right) W_{X}^{2}+\left(\frac{1}{2}-\sigma_{1}^{3}+2 \sigma_{1} \sigma_{2}\right) W_{X}^{3} \\
& +\left(\frac{1}{8}+\frac{1}{4} \sigma_{1}^{4}-3 \sigma_{1}^{2} \sigma_{2}+\sigma_{2}^{2}+2 \sigma_{1} \sigma_{3}\right) W_{X}^{4}, \\
I_{3}= & \left(\frac{1}{3}-\frac{2}{3} \sigma_{1}^{3}\right) W_{X}^{3}+\left(\frac{1}{2}+\sigma_{1}^{4}-2 \sigma_{1}^{2} \sigma_{2}\right) W_{X}^{4} .
\end{aligned}
$$

Inserting $I_{1}, I_{2}$, and $I_{3}$ into Eq. (A1) and comparing with Eq. (7) we obtain
Explicit expressions for the second- and third-order Poisson ratios, $\sigma_{2}$ and $\sigma_{3}$, in terms of elastic constants up to fourth order are available for cubic symmetry in Refs. 20 and 21. By using the isotropy relations for these elastic constants ${ }^{25} \sigma_{2}$ and $\sigma_{3}$ may be expressed in terms of the isotropic elastic constants up to fourth order as defined by Eq. (A1).

\section{APPENDIX B}

In order to solve

$$
u_{x x}-u_{x t}+[g(u)]_{x x}+u_{x x x t}=0 \text {, }
$$

with

$$
g(u)= \begin{cases}\frac{1}{2} u^{2}, & \text { quadratic case, IBE, } \\ \pm \frac{1}{3} u^{3}, & \text { cubic cases, MIBE, }\end{cases}
$$

numerically, a central difference approximation for the spatial and time derivatives is used. The resulting nine-point Crank-Nicolson system with a tridiagonal matrix is solved by Gauss elimination. The matrix has diagonal dominance. As a consequence the elimination procedure is stable. ${ }^{26}$

Discretization of (B1) with space step, $\Delta x$, and time step, $\Delta t$, in the quadratic case

$$
\begin{aligned}
-u_{p-1}^{q+1} & +\left[2+(\Delta x)^{2}\right] u_{p}^{q+1}-u_{p+1}^{q+1} \\
= & {\left[(\Delta t)^{2}-2\right]\left(u_{p+1}^{q}-2 u_{p}^{q}+u_{p-1}^{q}\right)+2(\Delta x)^{2} u_{p}^{q} } \\
& +\frac{1}{2}(\Delta t)^{2}\left[\left(u_{p+1}^{q}\right)^{2}-2\left(u_{p}^{q}\right)^{2}+\left(u_{p-1}^{q}\right)^{2}\right] \\
& +u_{p-1}^{q-1}-\left[2+(\Delta x)^{2}\right] u_{p}^{q-1}+u_{p+1}^{q-1}
\end{aligned}
$$


Flügge (Springer, Berlin, 1974), Vol. VI a/4, Mechanics of Solids IV, pp. 109-308.

${ }^{12}$ Reference 11, p. 222.

${ }^{13}$ L. Pochhammer, "Über die Fortpflanzungsgeschwindigkeiten kleiner Schwingungen in einem unbegrenztem isotropen Kreiszylinder," J. Rein Angew. Math. (Crelle) 81, 324-336 (1976).

${ }^{14} \mathrm{C}$. Chree, "The equations of an isotropic elastic solid in polar and cylindrical coordinates, their solutions and applications," Trans. Cambridge Philos. Soc. 14, 250-369 (1889).

is A. E. H. Love, A Treatise on the Mathematical Theory of Elasticity (Dover, New York, 1944), pp. 287-291.

${ }^{16}$ T. Hüter, "Über die Fortleitung von Ultraschallwellen in festen Stäben," Z. Angew Phys. 1, 274-289 (1949).

${ }^{17} \mathrm{M}$. Redwood and J. Lamb, "On the propagation of high frequency compressional waves in isotropic cylinders," Proc. Phys. Soc. London B 70, 136-143 (1957).

18J. R. Hutchinson and C. M. Percival, "Higher modes of longitudinal wave propagation in thin rods," J. Acoust. Soc. Am. 44, 1204-1210 (1968).
${ }^{19}$ A. E. Green and J. E. Adkins, Large Elastic Deformations (Clarendon, Oxford, UK, 1960).

${ }^{20} \mathrm{G}$. R. Barsch, "Higher order elastic constants and non-linear stress-strain

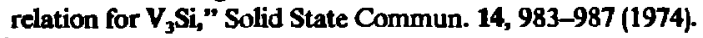

${ }^{21} \mathrm{U}$. Klein and F. Schwabl, "Ginzburg-Landau theory of coupled superconducting and magnetic phase transitions in A15 compounds," Physica 106B, 227-246 (1981).

${ }^{22} \mathrm{~T}$. Taniuti and C. C. Wei, “Reductive perturbation methods in non-linear wave propagation, I," J. Phys. Soc. Jpn. 24, $941-946$ (1968).

${ }^{23}$ Reference 1, p. 139.

${ }^{24}$ T. L. Perelman, A. Kh. Frieman, and M. M. El'Yashevich, "On the relationship between the $\mathrm{N}$-soliton solution of the modified Korteweg-de Vries equation and the KdV equation solution," Phys. Lett. 47A, 321-323 (1974).

29. A. A. Nranyan, "Higher order elastic constants of solids," Sov. Phys.Solid State 6, 1673-1675 (1965).

${ }^{26}$ W. A. Ames, Numerical Methods for Partial Differential Equations (Nelson, London, 1969), p. 47. 